\title{
EFFECT OF RANDOMLY FLUCTUATING ENVIRONMENT ON AUTOTROPH-HERBIVORE MODEL SYSTEM
}

\author{
TAPAN SAHA and MALAY BANDYOPADHYAY
}

Received 24 June 2004

\begin{abstract}
First we deal with a brief introduction of the autotroph-herbivore model system along with deterministic analysis of local stability, bifurcation behavior, and persistence of the populations. The second part consists of the stochastic formulation of the model system to incorporate the effect of environmental fluctuation and then analysis of nonequilibrium fluctuation. Stochastic stability criteria of the model system under the influence of random environmental fluctuation are obtained through the convergence conditions of second-order moments.
\end{abstract}

2000 Mathematics Subject Classification: 34F05, 60H10, 34A34, 92B05.

1. Introduction. The branch of science which deals with interrelationships among the living organisms in relation with the surrounding environment is known as ecology and an ecosystem is the functional unit of ecology. The interaction between living and nonliving (biotic or abiotic) components is a complex phenomenon. The remarkable variety of dynamical behaviors exhibited by many species of plants, insects, and animals has stimulated great interest in the development of mathematical models for several ecological systems [21, 23]. Mathematical modeling of ecological systems is a systematic methodology that has proved powerful as well as successful in discovering and better understanding the underlying processes and causes in nature based on its observable parts and their relationships. The purpose of a model is not to provide a literal description of some systems but to provide a conceptualization of the system and its workings, in terms of which one can think about the system and understand something of its behavior [22]. In other words modeling is frequently an evolving process. Systematic mathematical analysis can often lead to better understanding of the plausible models. The exposed discrepancies in turn lead to the necessary modifications [37].

The dynamical relationship between consumer and producer has long been and will continue to be one of the dominant themes in population biology due to its universal existence and importance [3, 6, 7, 9, 13]. All types of available literature deals with two types of autotroph-herbivore systems. These are terrestrial plant-grazer systems where the grazer is normally a mammal or insect, and aquatic or marine phytoplanktonzooplankton systems [3, 9, 10, 26, 28, 31]. Classical approaches for modeling plantherbivore systems have analogy with those for modeling the prey-predator systems. There is no typical pattern for the plant-herbivore dynamics; rather, it depends upon the demographic parameters of the plant and herbivore populations and also on the timing kind and degree of density dependence that they exhibit [25, 32]. A wide variety of 
modeling approaches have been used to reflect the quantitative diversity of the grazing systems. The dynamical problems involved with mathematical modeling of autotrophherbivore systems may appear to be simple at first sight; however, they may turn out to be complicated and challenging. In the last few decades, interest has been growing steadily in the designing and studying of mathematical models of autotroph-herbivore interactions.

Major parts of works in this direction are based on deterministic models of differential and difference equations. The deterministic approach has, however, some limitations in biology; it is always difficult to predict especially the future of the system accurately. This difficulty increases if we consider the complex behavior of the whole system or the dynamics of population ecosystems or global environmental systems. This type of difficulty arises as biological systems are controlled by environmental fluctuations. Randomness or stochasticity plays a vital role in the structure and function of biological systems. In ecology, we have two types of stochasticity-namely, the demographic stochasticity and the environmental stochasticity [12, 14, 24, 27]. Both types of stochasticity play a significant role in the realistic dynamic modeling of ecosystems [4]. A central obstacle in the stochastic modeling of an ecosystem is the lack of mathematical machinery available to analyze nonlinear multidimensional stochastic processes. Introduction of demographic or environmental stochasticity within a deterministic population model leads us to stochastic multispecies models. The resulting stochastic models involve nonlinear stochastic differential equations whose solutions pose great difficulties. Different linearization techniques for nonlinear stochastic differential equations generate a set of deterministic moment equations. These linearization techniques are receiving a great deal of attention not only in population biology but also in different fields of science and technology [2, 5, 19, 33]. In most of the cases randomness has been introduced in theoretical biology (mathematical biology and/or physical biology) by using the Gaussian white noise probably for the very reason that it gives some qualitative stochastic behavior of the model system without any complicated mathematical calculation [20].

The object of the present paper is to develop a stochastic dynamic model of a nonlinear noninteractive type of autotroph-herbivore model and to examine the stability conditions of the system under random perturbation. To make a comparative study of stability behaviors for the model system within both deterministic and stochastic environments, we have to find the criteria of stability within the deterministic as well as the stochastic environment. Section 2 consists of a brief introduction to the autotroph-herbivore model system and deterministic analysis of stability and Hopfbifurcation around the positive interior steady state of the model system. Section 3 includes the stochastic dynamic modeling of the autotroph-herbivore model system to include the effect of environmental fluctuation. The system of nonlinear stochastic differential equations has been reduced to deterministic moment equations with the help of statistical linearization in Section 4. Section 5 deals with the solutions for the system of second-order moment equations which leads us to the expressions for nonequilibrium fluctuation and the criteria of stability of the stationary state within stochastic environment. The paper ends with a concluding section. 
2. Autotroph-herbivore model: deterministic stability analysis. The consumerresource interactions can be classified into two independent directions: intimacy (the closeness and the duration of the relationship between the individual consumer and the organism it consumes) and lethality (the probability that a trophic interaction results in the death of the organism being consumed) [31, 35]. In case of autotroph-herbivore model system, herbivores are at a low scale of both "intimacy" and "lethality." It is a well-understood biological phenomenon that the changes in autotroph density affect the herbivores at both individual and population levels. Temporal variation in autotroph density affects the rate at which autotroph biomass are killed by herbivores. The most popular framework for modeling autotroph-herbivore interactions has the following structure:

$$
\begin{aligned}
& \frac{d N_{1}}{d t}=R\left(N_{1}\right) N_{1}-F\left(N_{1}, N_{2}\right) N_{2}, \\
& \frac{d N_{2}}{d t}=c F\left(N_{1}, N_{2}\right) N_{2}-\delta\left(N_{2}\right) N_{2},
\end{aligned}
$$

where $R\left(N_{1}\right)$ is the density-independent per capita growth rate of autotroph in absence of herbivores, $\delta\left(N_{2}\right)$ is the per capita decline rate of herbivores in the absence of autotrophs, $F\left(N_{1}, N_{2}\right)$ is the herbivores' functional response, and $c$ is the conversion rate of eaten autotroph biomass into new herbivores. The origin of (2.1) is clearly based on the classical Lotka-Volterra model, which is the simplest possible example of it (since it assumes exponential growth/decline terms and the linear functional response). The functional response is the rate at which each herbivore captures autotroph biomass. In the present study, we consider the Holling type-III functional response for herbivores. The type of behavior described by the Holling type-III function can easily occur if some fraction of the autotroph biomass is relatively well protected from consumption. The Holling type-III function has proved relatively successful in describing the feeding process of herbivores $[13,22]$. The dynamics of the model system is governed by the following system of nonlinear ordinary differential equations:

$$
\begin{gathered}
\frac{d N_{1}}{d t}=r N_{1}\left(1-\frac{N_{1}}{K}\right)-\frac{a N_{1}^{2} N_{2}}{b+N_{1}^{2}}, \\
\frac{d N_{2}}{d t}=\frac{c a N_{1}^{2} N_{2}}{b+N_{1}^{2}}-m N_{2},
\end{gathered}
$$

where $r, K, a, b, c, m$ are all positive constants and have the following biological significance: $r$ is the intrinsic growth rate of autotrophs in absence of herbivores, $K$ is the environmental carrying capacity of autotroph population, which is usually determined by the available sustaining resources, $a$ is the maximum uptake rate for herbivores associated with Holling type-III functional response, $b$ is the half-saturation autotroph density for type-III functional response, $c$ is the conversion efficiency, and $m$ is the mortality rate of herbivores in absence of autotroph. Now we assume that 
the conversion efficiency $c$ satisfies the condition $0<c<1$. Using the transformation $N_{1}=\sqrt{b} x, N_{2}=\sqrt{b} y$, and $t=K \tau / r \sqrt{b}$, (2.2) can be nondimensionalized as

$$
\begin{gathered}
\frac{d x}{d \tau}=x(\alpha-x)-\frac{\beta x^{2} y}{1+x^{2}}, \\
\frac{d y}{d \tau}=\frac{\beta_{1} x^{2} y}{1+x^{2}}-\gamma y,
\end{gathered}
$$

where $\alpha, \beta, \beta_{1}$, and $\gamma$ are dimensionless parameters and we have $0<\beta_{1}<\beta$. The initial condition for the system of (2.3) is given by $x(0)=x_{0} \geq 0$ and $y(0)=y_{0} \geq 0$ which are also biologically meaningful. Due to the boundedness of the functional responses and using (2.3) we can conclude that the functions on the right-hand side of differential equations (2.3) are continuous functions on $\mathbb{R}_{+}^{2}=[(x, y): x \geq 0, y \geq 0]$. Straightforward computation shows that they are Lipschitzian on $\mathbb{R}_{+}^{2}$. Hence the solutions of (2.3) with nonnegative initial condition exist and are unique. It is also easy to verify that these solutions exist for all $\tau>0$ and stay nonnegative. In fact, if $x(0)=x_{0}>0$, then $x(\tau)>0$ for all dimensionless time $\tau>0$. The same argument is true for the $y$ component. Hence, the interior of $\mathbb{R}_{+}^{2}$ is invariant under model system (2.3). Regarding the boundedness of solutions for the model system (2.3) with positive initial condition, we can state the following result.

LEMMA 2.1. All the solutions of the system (2.3) with $x(0)>0, y(0)>0$ are uniformly bounded within a region $B \subset \mathbb{R}_{+}^{2}$, where $\mathbf{B}=\left\{(x, y) \in \mathbb{R}_{+}^{2}: 0 \leq x \leq \alpha, 0 \leq x+\left(\beta / \beta_{1}\right) y \leq\right.$ $\left.L / \gamma, L=\alpha \gamma+\alpha^{2} / 4\right\}$.

The above lemma is very obvious; we omit its proof. Interested readers may proceed along the same way that we have adopted earlier [4].

The equilibrium points or steady states for the model system (2.3) are (i) $E_{0}(0,0)$ (trivial equilibrium point), (ii) $E_{1}(\alpha, 0)$ (axial equilibrium point), and (iii) $E_{*}\left(x^{*}, y^{*}\right)$ (positive interior equilibrium point), where

$$
x^{*}=\sqrt{\frac{\gamma}{\left(\beta_{1}-\gamma\right)}}, \quad y^{*}=\frac{\beta_{1}\left(\alpha-x^{*}\right)}{\left[\beta x^{*}\left(\beta_{1}-\gamma\right)\right]} .
$$

Hence the positive interior equilibrium $E_{*}$ will exist if and only if $\beta_{1}>\gamma$ and $0<x^{*}<\alpha$. For local stability of the model system around their steady states we have to find the Jacobian matrix for the system of (2.3). The Jacobian matrix for the system of (2.3) evaluated at any point $(x, y)$ is

$$
J(x, y)=\left[\begin{array}{cc}
\alpha-2 x-\frac{2 \beta x y}{\left(1+x^{2}\right)^{2}} & -\frac{\beta(x)^{2}}{1+\left(x^{2}\right)} \\
\frac{2 \beta_{1} x y}{\left(1+\left(x^{2}\right)\right)^{2}} & \frac{\beta_{1}\left(x^{2}\right)}{1+\left(x^{2}\right)}-\gamma
\end{array}\right] .
$$

At $E_{0}$, the Jacobian matrix takes the form

$$
[J(x, y)]_{E_{0}}=\left[\begin{array}{cc}
\alpha & 0 \\
0 & -\gamma
\end{array}\right] .
$$


The eigenvalues are $\lambda_{1}=\alpha>0$ and $\lambda_{2}=-\gamma<0$. Clearly $E_{0}$ is a saddle point which is unstable along the positive direction of the $x$-axis. At $E_{1}$, the Jacobian matrix takes the form

$$
[J(x, y)]_{E_{1}}=\left[\begin{array}{cc}
-\alpha & \frac{\beta(\alpha)^{2}}{1+(\alpha)^{2}} \\
0 & \frac{\beta_{1}(\alpha)^{2}}{1+(\alpha)^{2}}-\gamma
\end{array}\right]
$$

The eigenvalues are $\lambda_{1}=-\alpha$ and $\lambda_{2}=\beta_{1} \alpha /\left(1+(\alpha)^{2}\right)-\gamma$. Due to the condition $0<$ $x^{*}<\alpha$, we have $\lambda_{2}>0$. Thus $E_{1}$ is a saddle point which is unstable along the positive direction of the $y$-axis. At $E_{*}$ the Jacobian matrix takes the form

$$
[J(x, y)]_{E_{*}}=\left[\begin{array}{cc}
-\alpha-2 x^{*}-\frac{2 \beta x^{*} y^{*}}{\left(1+\left(x^{*}\right)^{2}\right)^{2}} & \frac{\beta\left(x^{*}\right)^{2}}{1+\left(x^{*}\right)^{2}} \\
\frac{2 \beta_{1} x^{*} y^{*}}{\left(1+\left(x^{*}\right)^{2}\right)^{2}} & 0
\end{array}\right]
$$

The characteristic equation for $J_{*}$ is

$$
\lambda^{2}+A_{1} \lambda+A_{2}=0
$$

where

$$
\begin{gathered}
A_{1}=-\operatorname{trace} J_{*}=-\left(\alpha-2 x^{*}-\frac{2 \beta x^{*} y^{*}}{\left(1+\left(x^{*}\right)^{2}\right)^{2}}\right), \\
A_{2}=\operatorname{det} J_{*}=\frac{2 \beta_{1} \beta\left(x^{*}\right)^{3} y^{*}}{\left(1+\left(x^{*}\right)^{2}\right)^{3}}>0 .
\end{gathered}
$$

Applying Routh-Hurwitz criteria to (2.9) we get the condition for local asymptotic stability of the steady-state $E_{*}$ as $A_{1}>0$, that is, $\alpha<2 x^{*} \gamma /\left(2 \gamma-\beta_{1}\right)$, with $\gamma<\beta_{1}<2 \gamma$. In this case $E_{*}$ is a global attractor in the positive $(x, y)$-plane. The stability behavior changes as $\alpha$ passes through the critical value $\alpha=\alpha^{*}=2 x^{*} \gamma /\left(2 \gamma-\beta_{1}\right)$ and the system exhibits Hopf-bifurcation. The existence condition for Hopf-bifurcation can be stated as follows.

LEMMA 2.2. If $\alpha=\alpha^{*}=2 x^{*} \gamma /\left(2 \gamma-\beta_{1}\right)$ with $\gamma<\beta_{1}<2 \gamma$, then the system (2.3) exhibits Hopf-bifurcation near $E_{*}$.

Proof. The lemma will be proved if we can show that the conditions for Hopfbifurcation are satisfied. At $\alpha=\alpha^{*}=2 x^{*} \gamma /\left(2 \gamma-\beta_{1}\right)$, $\operatorname{trace}\left(J_{*}\right)=0$ and $\operatorname{det}\left(J_{*}\right)=$ $2 \beta_{1} \beta\left(x^{*}\right)^{3} y^{*} /\left(1+\left(x^{*}\right)^{2}\right)^{3}>0$.

When $\alpha$ takes the value $\alpha=\alpha^{*}$, the two roots of the characteristic equation (2.9) are purely imaginary, namely, $\lambda= \pm i \omega$ where $\omega^{2}=\operatorname{det}\left(J_{*}\right)$ (evaluated at $\alpha=\alpha^{*}$ ). Also, we have

$$
\frac{d}{d \alpha}[\operatorname{trace}(J)]_{\alpha=\alpha^{*}}=\frac{2 \gamma-\beta_{1}}{\beta_{1}} \neq 0
$$


Hence all the conditions for Hopf-bifurcations are satisfied [16]. This ensures the existence of a small amplitude periodic solution near $E_{*}$.

We will now try to find the condition which eliminates the chance of existence of a nontrivial periodic solution within the bounded domain $B$. The approach is based on the application of the divergence criterion for the stability of periodic solutions in two-dimensional systems $[15,17,18]$.

We define the functions $h(x, y), f(x, y)$, and $g(x, y)$ as

$$
h(x, y)=\frac{1+x^{2}}{x^{2} y}, \quad f(x, y)=x(\alpha-x)-\frac{\beta x^{2} y}{1+x^{2}}, \quad g(x, y)=\frac{\beta_{1} x^{2} y}{1+x^{2}}-\gamma y
$$

Obviously $h(x, y)>0$ for $x, y>0$. Now,

$$
\Delta(x, y)=\frac{\partial}{\partial x}(h f)+\frac{\partial}{\partial y}(h g)=\alpha-2 x-\alpha x^{2}
$$

Thus $\Delta(x, y)<0$ for $\alpha^{1 / 3}<x<\alpha$ and by Bendixon-Dulac criterion there will be no limit cycle within $B$.

Recall that the saddle property of the boundary equilibrium point $E_{1}$ ensures the existence of the interior equilibrium point $E_{*}$. Local asymptotic stability of $E_{*}$ under the restrictions $\alpha<\alpha^{*}$ and $\gamma<\beta_{1}<2 \gamma$ together with nonexistence of trivial periodic solution (whenever $\alpha^{1 / 3}<x<\alpha$ ) implies global stability of the model system. Hence by using the arguments of Zhang et al. [38] we conclude that both populations will persist under the parametric restrictions mentioned earlier.

3. Autotroph-herbivore model: stochastic extension. Environmental fluctuation is an important component within an ecosystem. Most natural phenomena do not follow strictly deterministic laws; rather, they oscillate randomly about some average value so that the deterministic equilibrium is no longer an absolutely fixed state. May [22] pointed out the fact that due to environmental fluctuation, the birthrates, carrying capacity, competition coefficients, and other parameters involved with the model system exhibit random fluctuation to a greater or lesser extent. Consequently the equilibrium population distribution fluctuates randomly around some average values. Within the deterministic environment we seek the constant equilibrium populations and then investigate their stability which follows from the dynamics of the interactions between and within the species. For the systems which are driven by the environmental stochasticity, it is impossible to find a time-independent equilibrium point as a solution of the governing stochastic differential equations. In this situation it is reasonable to find a probabilistic "smoke cloud," described by the equilibrium probability distribution. The model systems is described by system of stochastic differential equations, there is a continuous spectrum of disturbances generated by the environmental stochasticity, and the system is in tension between two countervailing tendencies. On the one hand, the random environmental fluctuations are responsible for spreading the cloud, 
to make the probability distribution more diffusive, while, on the other hand, the dynamics of stabilizing population interactions tends to restore the populations to their mean value in order to compact the cloud. The model system with this type of compact cloud of population distribution is called a stochastically stable system. To study the effect of random environmental fluctuation we have to construct the stochastic counterpart of the deterministic model system by incorporating environmental fluctuation. Our next task is to extend the model system to a stochastic differential equation by incorporating additive white noise which will reflect the overall environmental effect on the model system.

Before introducing the stochastic perturbation we shift the origin to the positive interior equilibrium point $E_{*}\left(x^{*}, y^{*}\right)$ by using the transformation $x=x^{*}+p, y=$ $y^{*}+h$. Substituting this transformation in (2.3) and using Taylor series expansion for the functions on the right-hand sides of (2.3) we get

$$
\begin{gathered}
\frac{d p}{d \tau}=a_{10} p+a_{01} h+a_{20} p^{2}+a_{11} p h, \\
\frac{d h}{d \tau}=b_{10} p+b_{20} p^{2}+b_{11} p h .
\end{gathered}
$$

The system of (3.1) are basic deterministic ordinary differential equations governing the system behavior around the steady state $E_{*}$. In the above expansion, we have discarded the third and higher powers of the perturbation variables $p$ and $h$ as the deterministic and stochastic stability properties of the model system near the origin solely depend upon the coefficients of first- and second-order homogeneous terms with the variables $p$ and $h[2,4,5]$. Coefficients involved in (3.1) are given by

$$
\begin{gathered}
a_{10}=\frac{\alpha\left(2 \gamma-\beta_{1}\right)-2 x^{*} \gamma}{\beta_{1}}, \quad a_{01}=-\frac{\beta \gamma}{\beta_{1}}, \quad a_{11}=-\frac{2 \beta\left(\beta_{1}-\gamma\right)^{2}}{\beta_{1}^{2}}, \\
a_{20}=\frac{\gamma\left(\alpha-x^{*}\right)(5 \beta-4 \gamma)}{\beta_{1}^{2} x^{*}}-\alpha \beta_{1}^{2}, \quad b_{10}=\frac{2\left(\beta_{1}-\gamma\right)^{2} x^{*} y^{*}}{\beta_{1}}, \\
b_{11}=\frac{2\left(\beta_{1}-\gamma\right)^{2} x^{*}}{\beta_{1}}, \quad b_{20}=\frac{\gamma^{*}\left(\beta_{1}-4 \gamma\right)\left(\beta_{1}-\gamma\right)^{2}}{\beta_{1}^{2}} .
\end{gathered}
$$

In particular, as we are interested in stochastic stability of the model system under environmental fluctuation (within the vicinity of the origin) in terms of second-order moments, terms involving third and higher powers will have no contribution to the stability behavior. The solutions $(p(\tau), h(\tau))$ of (3.1) subject to known nonnegative initial values represent the state of the system at any future time $\tau>0$. If the random aspects of the system are to be concerned, a number of modifications can be made in the formation of the initial value problem given by (3.1). We will however follow the case when imposing random input or perturbation extends the right-hand side of (3.1) to a system of stochastic differential equations. More specifically we are concerned with stochastic differential equations which are driven by Gaussian white noises and interpreted 
mathematically as Ito stochastic differential equations. Gaussian white noise, which is a delta-correlated random process, is very irregular and as such it is to be treated carefully. In spite of this it is an immensely useful concept to model rapidly fluctuating environments. White noises are a very good approximation of many phenomena of physical and natural systems such as electrical resistance, motion of Brownian particles, climate fluctuation an so forth, and support the usefulness of the white noises idealization in application to ecological systems. To study the effect of environmental fluctuations on the stability properties of the steady state, the system of (3.1) can be extended to Ito-type stochastic differential equations (nonlinear coupled bivariate Langevin equations) by introducing additive white noises as follows:

$$
\begin{gathered}
\frac{d p}{d \tau}=a_{10} p+a_{01} h+a_{20} p^{2}+a_{11} p h+\chi_{1}(\tau), \\
\frac{d h}{d \tau}=b_{10} p+b_{20} p^{2}+b_{11} p h+\chi_{2}(\tau),
\end{gathered}
$$

where $\chi_{1}(\tau)$ and $\chi_{2}(\tau)$ are Gaussian white noises characterized by $\left\langle\chi_{1}(\tau)\right\rangle=0=$ $\left\langle\chi_{2}(\tau)\right\rangle$ and $\left\langle\chi_{i}(\tau) \chi_{j}\left(\tau_{1}\right)\right\rangle=2 \xi \delta_{i j} \delta\left(\tau-\tau_{1}\right)(i, j=1,2)$, where $\xi$ is the intensity or strength of the random perturbation, $\delta_{i j}$ is the Dirac delta function, with $\tau$ and $\tau_{1}$ being the distinct times, and the bracket $\langle\cdot\rangle$ represents the ensemble average. Equations (3.3) are the basic nonlinear stochastic differential equations determining the statistical behavior of the system around the steady-state $E_{*}$.

4. Statistical linearization and moment equations. The statistical linearization of the system of equations consists of replacing (3.3) by the system of linear equations [36]

$$
\begin{aligned}
& \frac{d p}{d \tau}=\zeta_{1} p+\eta_{1} h+\phi_{1}+\chi_{1}(\tau) \\
& \frac{d p}{d \tau}=\zeta_{2} p+\eta_{2} h+\phi_{2}+\chi_{2}(\tau) .
\end{aligned}
$$

The errors committed due to this linearization are given by

$$
\begin{gathered}
\Delta_{1}(p, h)=\left(a_{10} p+a_{01} h+a_{20} p^{2}+a_{11} p h\right)-\left(\zeta_{1} p+\eta_{1} h+\phi_{1}\right), \\
\Delta_{2}(p, h)=\left(b_{10} p+b_{20} p^{2}+b_{11} p h\right)-\left(\zeta_{2} p+\eta_{2} h+\phi_{2}\right) .
\end{gathered}
$$

The unknown coefficients $\zeta_{1}, \zeta_{2}, \eta_{1}, \eta_{2}, \phi_{1}, \phi_{2}$ of (4.1) are determined from the minimization of the averages of the squares of the errors given by (4.2). We determine the unknown coefficients by demanding that

$$
\frac{\partial}{\partial \zeta_{i}}\left\langle\Delta_{i}^{2}\right\rangle=\frac{\partial}{\partial \eta_{i}}\left\langle\Delta_{i}^{2}\right\rangle=\frac{\partial}{\partial \phi_{i}}\left\langle\Delta_{i}^{2}\right\rangle=0,
$$


where $i=1,2$ and $\langle\cdot\rangle$ represents the ensemble average. The expressions for $\zeta_{i}, \eta_{i}, \phi_{i}$ $(i=1,2)$ are given by

$$
\begin{array}{lll}
\zeta_{1}=a_{10}, & \eta_{1}=a_{01}, & \phi_{1}=a_{20}\left\langle p^{2}\right\rangle+a_{11}\langle p h\rangle, \\
\zeta_{2}=b_{10}, & \eta_{2}=0, & \phi_{2}=b_{20}\left\langle p^{2}\right\rangle+b_{11}\langle p h\rangle .
\end{array}
$$

After evaluating the unknown coefficients, simple calculations lead to the system of ordinary differential equations for first two moments

$$
\begin{gathered}
\frac{d\langle p\rangle}{d \tau}=a_{10}\langle p\rangle+a_{01}\langle h\rangle+a_{20}\left\langle p^{2}\right\rangle+a_{11}\langle p h\rangle, \\
\frac{d\langle h\rangle}{d \tau}=b_{10}\langle p\rangle++b_{20}\left\langle p^{2}\right\rangle+b_{11}\langle p h\rangle, \\
\frac{d\left\langle p^{2}\right\rangle}{d \tau}=2 a_{10}\left\langle p^{2}\right\rangle+2 a_{01}\langle p h\rangle+2 a_{20}\left\langle p^{3}\right\rangle+2 a_{11}\left\langle p^{2} h\right\rangle+2 \epsilon_{1}, \\
\frac{d\left\langle h^{2}\right\rangle}{d \tau}=2 b_{10}\langle p h\rangle+2 b_{20}\left\langle p^{2} h\right\rangle+2 b_{11}\left\langle p h^{2}\right\rangle+2 \epsilon_{2}, \\
\frac{d\langle p h\rangle}{d \tau}=b_{10}\left\langle p^{2}\right\rangle+a_{01}\left\langle h^{2}\right\rangle+a_{10}\langle p h\rangle+\left(a_{20}+b_{11}\right)\left\langle p^{2} h\right\rangle+b_{20}\left\langle p^{3}\right\rangle+a_{11}\left\langle p h^{2}\right\rangle,
\end{gathered}
$$

where we have used the results

$$
\left\langle p \chi_{1}\right\rangle=\epsilon_{1}, \quad\left\langle p \chi_{2}\right\rangle=0, \quad\left\langle h \chi_{1}\right\rangle=0, \quad\left\langle h \chi_{2}\right\rangle=\epsilon_{2} .
$$

We assume that the system size expansion is valid such that all correlations and variances are of the order of $1 / N$ compared to the averages $[2,4,5,30]$, that is,

$$
\langle p h\rangle \propto o\left[\frac{\langle p\rangle}{N}\right] \quad \text { or } \quad o\left[\frac{\langle h\rangle}{N}\right], \quad\left\langle p^{2}\right\rangle \propto o\left[\frac{\langle p\rangle}{N}\right], \quad\left\langle h^{2}\right\rangle \propto o\left[\frac{\langle h\rangle}{N}\right],
$$

where $N$ is the total population size of the system. We also assume that the correlations $\epsilon_{1}$ and $\epsilon_{2}$ given by (4.7) decrease with the increase of the population size and they are assumed to be of the order of the inverse of the total population size $N$. To calculate these moments, we now express $\left\langle p^{2} h\right\rangle,\left\langle p h^{2}\right\rangle,\left\langle p^{3}\right\rangle$ in terms of the first two moments for each of the variables and the correlation coefficients using a bivariate Gaussian distribution. Since we are interested only in the first two moments, it is convenient to use the characteristic function

$$
\psi\left(\nu_{1}, \nu_{2}\right)=\exp \left[i\langle p\rangle \nu_{1}+i\langle h\rangle \nu_{2}-\frac{1}{2}\left(\sigma_{1}^{2} v_{1}^{2}+\sigma_{2}^{2} \nu_{2}^{2}+2 \rho_{12} \sigma_{1} \sigma_{2} \nu_{1} \nu_{2}\right)\right]
$$

where

$$
\begin{aligned}
\sigma_{1}^{2} & =\left\langle p^{2}\right\rangle-(\langle p\rangle)^{2}, \\
\sigma_{2}^{2} & =\left\langle h^{2}\right\rangle-(\langle h\rangle)^{2}, \\
\rho_{12} & =\frac{\langle p h\rangle-\langle p\rangle\langle h\rangle}{\sigma_{1} \sigma_{2}} ;
\end{aligned}
$$


using the result [36]

$$
\left\langle p^{n} h^{m}\right\rangle=\left[(-i)^{(m+n)} \frac{\partial^{n+m}}{\partial v_{1}^{n} \partial v_{2}^{m}}\left[\psi\left(\nu_{1}, \nu_{2}\right)\right]\right]_{\left(v_{1}=v_{2}=0\right)}
$$

one can find the following results:

$$
\begin{gathered}
\left\langle p^{2} h\right\rangle=2\langle p\rangle[\langle p h\rangle-\langle p\rangle\langle h\rangle]+\left\langle p^{2}\right\rangle\langle h\rangle, \\
\left\langle p h^{2}\right\rangle=2\langle h\rangle[\langle p h\rangle-\langle p\rangle\langle h\rangle]+\langle p\rangle\left\langle h^{2}\right\rangle, \\
\left\langle p^{3}\right\rangle=3\langle p\rangle\left\langle p^{2}\right\rangle-2(\langle p\rangle)^{3} .
\end{gathered}
$$

Thus using the above expression, keeping the lowest-order terms, and replacing the averages $\langle p\rangle$ and $\langle h\rangle$ by their steady-state values $\langle p\rangle=0=\langle h\rangle$, we get the following reduced system of equations:

$$
\begin{gathered}
\frac{d\left\langle p^{2}\right\rangle}{d \tau}=2 a_{10}\left\langle p^{2}\right\rangle+2 a_{01}\langle p h\rangle, \\
\frac{d\left\langle h^{2}\right\rangle}{d \tau}=2 b_{10}\langle p h\rangle, \\
\frac{d\langle p h\rangle}{d \tau}=b_{10}\left\langle p^{2}\right\rangle+a_{01}\left\langle h^{2}\right\rangle+a_{10}\langle p h\rangle .
\end{gathered}
$$

These equations are the required ordinary coupled linear equations for second-order moments.

5. Stochastic stability analysis. Stability of the model system in presence of environmental fluctuation demands that the second-order moments approach to zero or a constant value with the advancement of time [1, 4, 12]. For this purpose we have to study the nature of the solution of the system of linear differential equations for second-order moments. Eliminating $\left\langle h^{2}\right\rangle$ and $\left\langle p^{2}\right\rangle$ from the system of (4.12) we get an ordinary linear differential equation for $\langle p h\rangle$ as

$$
\frac{d^{3}}{d \tau^{3}}\langle p h\rangle+3 u_{1} \frac{d^{2}}{d \tau^{2}}\langle p h\rangle+3 u_{2} \frac{d}{d \tau}\langle p h\rangle+u_{3}\langle p h\rangle=0
$$

where

$$
u_{1}=-a_{10}, \quad 3 u_{2}=2 a_{10}^{2}-4 a_{01} b_{10}, \quad u_{3}=4 a_{01} a_{10} b_{10}
$$

Assuming the solution of (5.1) in the form $\langle p h\rangle=A \exp (\mu \tau)$ and then substituting, we get the auxiliary equation

$$
\mu^{3}+3 u_{1} \mu^{2}+3 u_{2} \mu+u_{3}=0
$$


The structure and nature of the solution of the system of (4.12) solely depends upon the sign of discriminating cubic " $G$ " of the cubic equation (5.3) and is given by $3 G=$ $a_{10}^{2}+4 a_{01} b_{10}$. First we consider the case $G<0$. Then the roots of the auxiliary equation of (5.3) are given by $-u_{1},-u_{1} \pm+i \sqrt{3 G_{1}}$, where $G_{1}=-G$. The solution of the linear differential equation (5.1) and hence the system of linear differential equations (4.12) given by

$$
\begin{gathered}
\langle p h\rangle=\exp \left(-u_{1} \tau\right)\left[M_{1}+M_{2} \cos \sqrt{3 G_{1}} \tau+M_{3} \sin \sqrt{3 G_{1}} \tau\right] \\
\left\langle p^{2}\right\rangle=\exp \left(-u_{1} \tau\right)\left[N_{1}+N_{2} \cos \sqrt{3 G_{1}} \tau+N_{3} \sin \sqrt{3 G_{1}} \tau\right]+N_{4} \exp \left(-2 u_{1} \tau\right), \\
\left\langle h^{2}\right\rangle=\exp \left(-u_{1} \tau\right)\left[P_{1}+P_{2} \cos \sqrt{3 G_{1}} \tau+p_{3} \sin \sqrt{3 G_{1}} \tau\right]+P_{4},
\end{gathered}
$$

where $M_{i}(i=1,2,3), N_{i}, P_{i}(i=1,2,3,4)$ are all constants. The convergence of the second-order moments depends solely on the sign of $u_{1}$. For $u_{1}>0$, the system under stochastic perturbation is stable in the sense of second-order moments around the equilibrium state $E_{*}$ and is unstable for $u_{1}<0$ [1].

On the other hand, for $G>0$, the roots of the auxiliary equation of (5.3) are given by $-u_{1},-u_{1} \pm \sqrt{3 G}$. The solutions of (4.12) are given by

$$
\begin{gathered}
\langle p h\rangle=\exp \left(-u_{1} \tau\right)\left[Q_{1}+Q_{2} \exp (-\sqrt{3 G} \tau)+Q_{3} \exp (\sqrt{3 G} \tau)\right], \\
\left\langle p^{2}\right\rangle=\exp \left(-u_{1} \tau\right)\left[R_{1}+R_{2} \exp (-\sqrt{3 G} \tau)+R_{3} \exp (\sqrt{3 G} \tau)\right]+R_{4} \exp \left(-2 u_{1} \tau\right), \\
\left\langle h^{2}\right\rangle=\exp \left(-u_{1} \tau\right)\left[S_{1}+S_{2} \exp (-\sqrt{3 G} \tau)+S_{3} \exp (\sqrt{3 G} \tau)\right]+S_{4},
\end{gathered}
$$

where $Q_{i}(i=1,2,3), R_{i}, S_{i}(i=1,2,3,4)$ are all constants. Hence in this case the convergence of the stochastic model system in terms of second-order moment demands $u_{1}>0$ along with $u_{1}>\sqrt{3 G}$. For $u_{1}<0$ or $u_{1}<\sqrt{3 G}$, fluctuation about the steady states increases with the increase of time $t$ and hence the second-order moments diverge with the advancement of time; accordingly the system becomes unstable in the sense of second-order moments [1]. In case of deterministic analysis, local asymptotic stability of the steady-state $E_{*}$ demands the criteria $\alpha<2 x^{*} \gamma /\left(2 \gamma-\beta_{1}\right)=\alpha^{*}$, with $2 \gamma>\beta_{1}$, which is nothing but $u_{1}>0$. But for the stochastic version of the model system, the stability of the steady state demands an additional restriction $u_{1}>\sqrt{3 G}$. The above analysis leads us to an important conclusion that $u_{1}=\sqrt{3 G}$ is a critical parametric condition for which the stochastic model system changes its stability behavior around the equilibrium point $E_{*}$. Thus $u_{1}=\sqrt{3 G}$ or equivalently $\alpha=\alpha^{* *}=\sqrt{\gamma /\left(\beta_{1}-\gamma\right)}$ is the new bifurcation point and the stability behavior of the stochastic model system under environmental fluctuation changes as $u_{1}$ passes through the value $\sqrt{3 G}$ from lower to higher.

6. Conclusion. In the present paper, we have considered a noninteractive type of autotroph-herbivore model system in which the removal rate of autotroph biomass has been taken as a Holling type-III function and we have derived the local asymptoticstability condition and existence of small amplitude periodic solution within deterministic environment. It also includes the stochastic dynamical aspects of stability of 
the model system within a fluctuating environment with special emphasis on the comparative study of stability results in two different environments.

The type of behavior described by the Holling type-III function can easily occur if some fraction of the resource biomass is well protected from consumption [11]. This type of uptake function also reflects the idea that autotroph biomass in low abundances is harder to find and capture by the herbivores. The existence of the positive interior equilibrium point and its local stability is totally controlled by the environmental carrying capacity of autotroph biomass and rate of change (or increase) of herbivore biomass due to herbivory compared to their mortality rate [29, 30]. Local asymptotic stability of coexisting equilibrium points demands that the carrying capacity of autotroph biomass be greater than some threshold value and that the rate of consumption of autotroph biomass lie between a finite bounded interval along with the mortality rate of herbivore biomass must be less than the growth rate of herbivores due to herbivory. All these ecologically significant results are the interpretations of various restrictions we have obtained in Section 2. Conditions for global stability and permanence of solutions indicate the longtime survival of both species.

Deterministic models in ecology do not usually incorporate environmental fluctuation based upon the idea that in the case of large populations, stochastic deviations (or effects of randomly fluctuating environment) are small enough to be ignored [8, 34]. In reality, the stochastic model provides a more realistic picture of a natural system than its deterministic counterpart. To analyze the stability properties of the model system embedded in a fluctuating environment around its coexisting equilibrium point, we have considered the stochastic differential equation model of autotroph-herbivore populations. The deterministic model is randomized by introducing additive Gaussian white noise leading to a system of Ito type of stochastic differential equation. By using a minimization of the averages of error square, the resulting system is linearized and its stability condition is obtained by means of the corresponding second-order moments. The point of importance is that the linearization is achieved by a Galerkinlike technique instead of standard Taylor expansion. Basically, the stochastic stability is converted into a deterministic stability by using the dynamical equation of the state moment. It is also possible to follow the route of stochastic Liapunov function to obtain the stability condition but it is not a suitable one for the bifurcation analysis. Stochastic stability of the model system demands some extra restrictions on the parameters than the deterministic case. Our analysis reveals the fact that lowering of environmental carrying capacity and strong interaction between autotroph-herbivore population drives the system towards instability within the fluctuating environment. Environmental fluctuation is also responsible for the shift of bifurcation point from $\alpha=\alpha^{*}$ to the new bifurcation point $\alpha=\alpha^{* *}$. These are in good agreement with some earlier results $[26,29,30]$.

ACKNOWLEDGMENTs. The authors wish to thank Professor C. G. Chakrabarti, an S. N. Bose Professor of theoretical physics, Department of Applied Mathematics, for his valuable suggestions, which helped in better exposition of the paper. The first author wishes to thank the University Grants Commission of India for its Junior Research fellowship. 


\section{REFERENCES}

[1] L. Arnold, Stochastic Differential Equations: Theory and Applications, Wiley-Interscience, New York, 1974.

[2] M. C. Baishya and C. G. Chakraborti, Nonequilibrium fluctuation in Volterra-Lotka systems, Bull. Math. Biol. 49 (1987), no. 1, 125-131.

[3] M. Bandyopadhyay, R. Bhattacharyya, and B. Mukhopadhyay, Dynamics of an autotrophherbivore ecosystem with nutrient recycling, Eco. Mod. 176 (2004), 201-209.

[4] M. Bandyopadhyay and C. G. Chakrabarti, Deterministic and stochastic analysis of a nonlinear prey-predator systems, J. Biol. Systems 11 (2003), no. 2, 161-172.

[5] S. Banerjee and C. G. Chakrabarti, Stochastic dynamic modelling of damped Lotka-Volterra system, Systems Anal. Modelling Simulation 30 (1998), no. 1-2, 1-10.

[6] E. Beretta and Y. Kuang, Global analyses in some delayed ratio-dependent predator-prey systems, Nonlinear Anal. 32 (1998), no. 3, 381-408.

[7] A. A. Berryman, The origin and evolution of predator-prey theory, Ecology 73 (1992), 15301535.

[8] M. Carletti, On the stability properties of a stochastic model for phage-bacteria interaction in open marine environment, Math. Biosci. 175 (2002), no. 2, 117-131.

[9] G. Caughley, Plant-herbivore systems, Theoretical Ecology: Principles and Applications (R. M. May, ed.), W. B. Saunders, Pennsylvania, 1976, pp. 94-113.

[10] M. J. Crawley, Herbivory. The Dynamics of Animal-Plant Interactions, University of California Press, California, 1983.

[11] D. L. DeAngelis, Dynamics of Nutrient Cycling and Food Webs, Chapman \& Hall, London, 1992.

[12] C. W. Gardiner, Handbook of Stochastic Methods, Springer Series in Synergetics, vol. 13, Springer-Verlag, Berlin, 1983.

[13] D. Ghosh and A. K. Sarkar, Oscillatory behavior of an autotroph-herbivore system with a type-III uptake function, Internat. J. Systems Sci. 28 (1997), no. 3, 259-264.

[14] W. S. C. Gurney and R. M. Nisbet, Ecological Dynamics, Oxford University Press, New York, 1998.

[15] J. K. Hale, Ordinary Differential Equations, Robert E. Krieger Publishing, New York, 1980.

[16] B. D. Hassard, N. D. Kazarinoff, and Y. H. Wan, Theory and Applications of Hopf Bifurcation, London Mathematical Society Lecture Note Series, vol. 41, Cambridge University Press, Cambridge, 1981.

[17] J. Hofbauer and K. Sigmund, The Theory of Evolution and Dynamical Systems, London Mathematical Society Student Texts, vol. 7, Cambridge University Press, Cambridge, 1988.

[18] S. B. Hsu, On global stability of a predator-prey system, Math. Biosci. 39 (1978), no. 1-2, $1-10$.

[19] G. Jumarie, A practical variational approach to stochastic optimal control via state moment equations, J. Franklin Inst. B 332 (1995), no. 6, 761-772.

[20] - Stochastics of order $n$ in biological systems: applications to population dynamics, thermodynamics, nonequilibrium phase and complexity, J. Biol. Systems 11 (2003), no. 2, 113-137.

[21] M. Kot, Elements of Mathematical Ecology, Cambridge University Press, Cambridge, 2001.

[22] R. M. May, Stability and Complexity in Model Ecosystems, Princeton University Press, New Jersey, 2001.

[23] J. D. Murray, Mathematical Biology, Biomathematics, vol. 19, Springer-Verlag, Berlin, 1993.

[24] R. M. Nisbet and W. S. C. Gurney, Modelling Fluctuating Populations, Wiley-Interscience, 1982.

[25] S. Pimm, Food Webs, Chapman \& Hall, London, 1982.

[26] Prajneshu, A stochastic model for two interacting species, Stochastic Process. Appl. 4 (1976), no. 3, 271-282. 
[27] E. Renshaw, Modelling Biological Populations in Space and Time, Cambridge Studies in Mathematical Biology, vol. 11, Cambridge University Press, Cambridge, 1991.

[28] S. G. Ruan, Persistence and coexistence in zooplankton-phytoplankton-nutrient models with instantaneous nutrient recycling, J. Math. Biol. 31 (1993), no. 6, 633-654.

[29] G. P. Samanta, Influence of environmental noises on the Gomatam model of interacting species, Eco. Mod. 91 (1996), no. 1, 283-291.

[30] G. P. Samanta and C. G. Chakrabarti, Stochastic behaviour of a damped Volterra-Lotka system with time-dependent parameters, J. Math. Phys. Sci. 25 (1991), no. 4, 351359.

[31] P. Stiling, Ecology: Theories and Applications, Prentice-Hall, New York, 1999.

[32] M. Stubbs, Density dependence in the life cycles of animals and its importance in $k$-and $r$-strategies, J. Animal Ecology 46 (1977), no. 2, 677-688.

[33] Yu. M. Svirezhev and D. O. Logofet, Stability of Biological Communities, Mir, Moscow, 1983.

[34] P. K. Tapaswi and A. Mukhopadhyay, Effects of environmental fluctuation on plankton allelopathy, J. Math. Biol. 39 (1999), no. 1, 39-58.

[35] P. Turchin, Complex Population Dynamics. A Theoretical/Empirical Synthesis, Monographs in Population Biology, vol. 35, Princeton University Press, New Jersey, 2003.

[36] M. C. Valsakumar, K. P. N. Murthy, and G. Ananthakrishna, On the linearization of nonlinear Langevin-type stochastic differential equations, J. Statist. Phys. 30 (1983), 617-631.

[37] K. Yang, Basic properties of mathematical population models, J. Biomath. 17 (2002), no. 2, 129-142.

[38] X. Zhang, L. Chen, and A. U. Neumann, The stage-structured predator-prey model and optimal harvesting policy, Math. Biosci. 168 (2000), no. 2, 201-210.

Tapan Saha: Department of Applied Mathematics, University of Calcutta, 92 A.P.C. Road, Calcutta 700009, India

E-mail address: tsmath@rediffmai 1 .com

Malay Bandyopadhyay: Department of Mathematics, Scottish Church College, 1 \& 3 Urquhart Square, Calcutta 700006, India

E-mail address: ma1ay_ban@rediffmai 1.com 


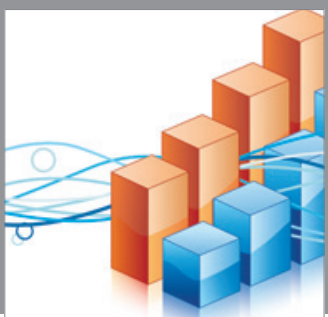

Advances in

Operations Research

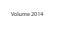

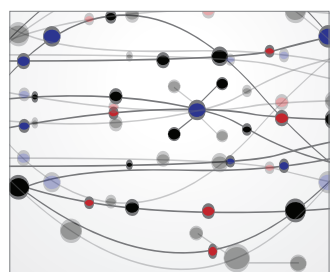

\section{The Scientific} World Journal
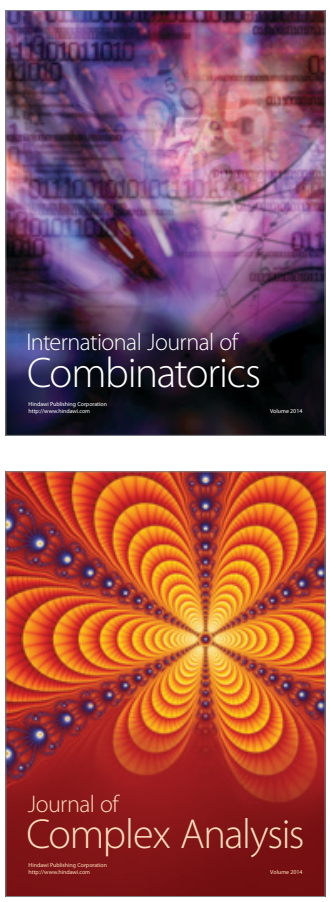

International Journal of

Mathematics and

Mathematical

Sciences
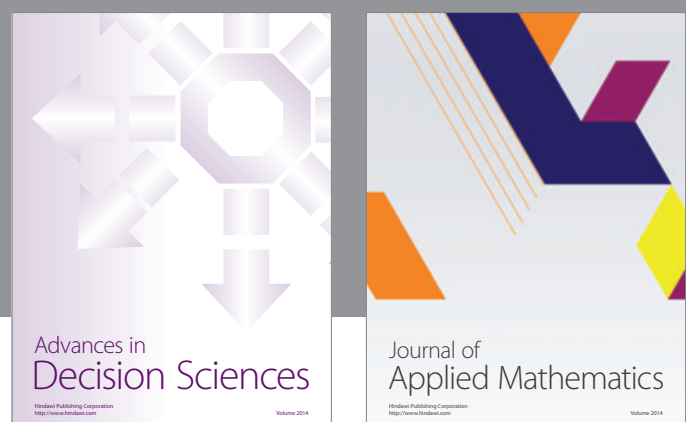

Journal of

Applied Mathematics
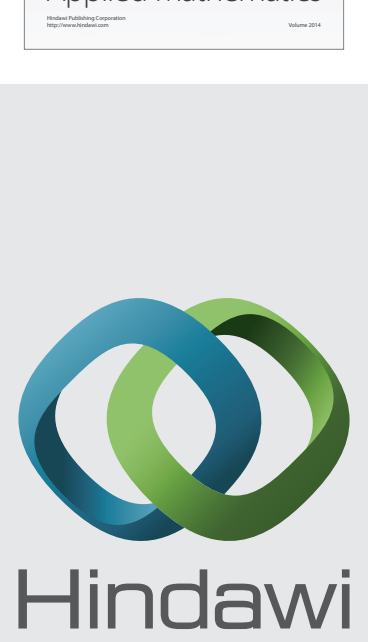

Submit your manuscripts at http://www.hindawi.com
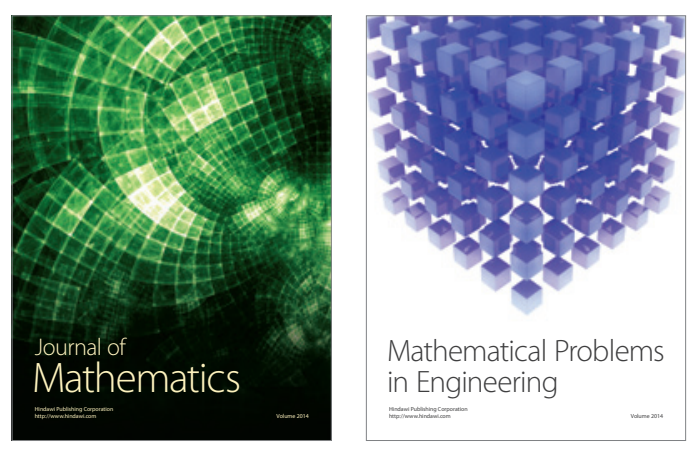

Mathematical Problems in Engineering
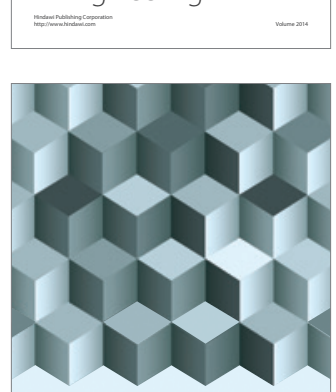

Journal of

Function Spaces
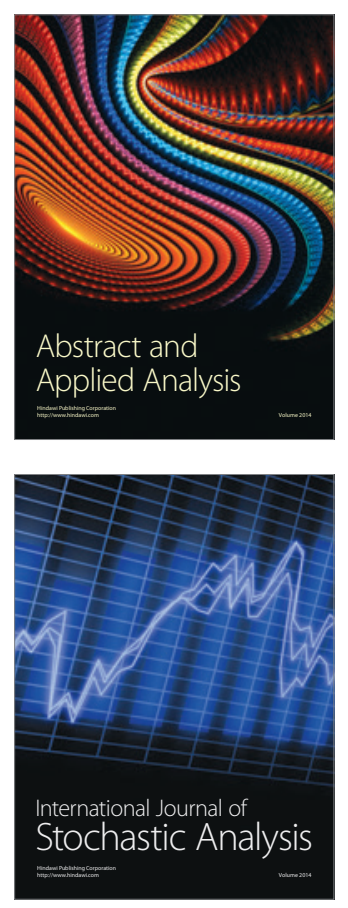

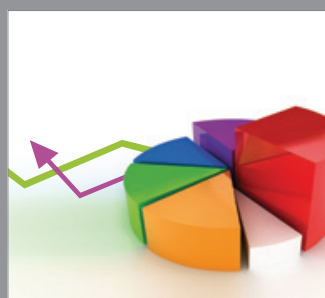

ournal of

Probability and Statistics

Promensencen
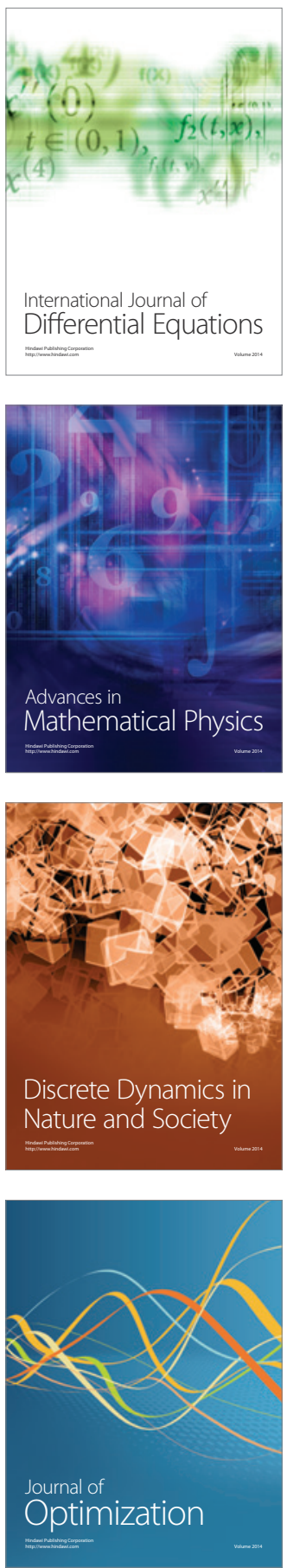